\title{
UM OLHAR HOLÍSTICO SOBRE A QUÍMICA DE PRODUTOS NATURAIS BRASILEIRA
}

\author{
Angelo C. Pinto* e Claudia M. Rezende \\ Instituto de Química, Universidade Federal do Rio de Janeiro, CT, B1 A, 21945-970 Cidade Universitária, Rio de Janeiro - RJ \\ Fernanda R. Garcez \\ Departamento de Química, Centro de Ciências Exatas e Tecnológica, Universidade Federal de Mato Grosso do Sul, 79070-900 \\ Campo Grande - MS \\ Rosângela de A. Epifanio \\ Instituto de Química, Universidade Federal Fluminense, Campus do Valonguinho, 24210-005 Niterói - RJ
}

\begin{abstract}
AN OVERVIEW OF THE BRAZILIAN NATURAL PRODUCT COMMUNITY. This paper describes a comparative analysis on natural product chemistry between the Brazilian Chemical Society journals and twelve representative international publications in the area. The search using the ISI Web of Knowledge disclosed 41,362 publications in the years 2000-2002 containing at least one researcher from a Brazilian Institution, from which $12 \%$ belongs to natural products.
\end{abstract}

Keywords: Natural Product Chemistry; Química Nova; Journal of the Brazilian Chemical Society.

\section{INTRODUÇÃO}

A criação dos periódicos Química Nova (QN) e Journal of the Brazilian Chemical Society (JBCS) é um dos marcos históricos da química brasileira ${ }^{1}$.

A história da ciência brasileira mostra, entretanto, que as revistas científicas brasileiras têm vida efêmera. Essa afirmativa pode ser generalizada para todas as revistas científicas dos países da América do Sul e da América Central ${ }^{2}$. São muitas as razões para essa efemeridade e não cabe aqui enumerá-las, porque isso foge ao escopo deste trabalho. Uma das principais razões é a falta de compromisso da comunidade científica brasileira com as suas revistas, talvez até porque não acredite na sua qualidade ou porque prefira ver seus artigos científicos veiculados em periódicos estrangeiros. São exceções a essa regra a Revista do Instituto Histórico e Geográfico Brasileiro que publica regularmente desde a sua fundação, em 1838 , discussões de natureza política e cultural em torno de questões da organização social, cujo objetivo principal é apresentar o pensamento e a história nacional ${ }^{3}$, e os Anais da Academia Brasileira de Ciências. A outra exceção é a Revista científica do Instituto Oswaldo Cruz (Memórias do Instituto Oswaldo Cruz), um dos 21 periódicos brasileiros indexados ao Institute of Scientific Information (ISI), fundada em 1909 graças aos ideais visionários do grande cientista brasileiro Oswaldo Cruz. Esta é hoje uma das publicações mais importante da América Latina. As Memórias do Instituto Oswaldo Cruz, publicam trabalhos nas áreas de parasitologia (incluindo protozoologia, helmintologia, entomologia e malacologia), microbiologia (incluindo virologia, bacteriologia e micologia) e medicina tropical (incluindo patologia, epidemiologia e estudos clínicos), além de estudos básicos em bioquímica, imunologia, biologia celular e molecular, fisiologia, farmacologia e genética relacionados a essas áreas. Sua versão "on-line" pode ser encontrada no endereço http://memorias.ioc.fiocruz.br.

O objetivo deste artigo é avaliar o que vem sendo publicado no Journal of the Brazilian Chemical Society (JBCS) e na Química Nova (QN) na área de produtos naturais (PN), a sub-área da química mais antiga e consolidada no País ${ }^{4}$. Essa avaliação tem, entre as suas finalidades, o propósito de incentivar a comunidade de PN a publicar no

*e-mail: angelo@iq.ufrj.br
JBCS e na QN os resultados de suas pesquisas. Só assim se garantirá a perenidade e a visibilidade destes dois periódicos. O que, em princípio, deveria ser uma tarefa simples, sempre foi e continua a ser uma das razões principais da vida efêmera das revistas científicas brasileiras, porque os cientistas brasileiros preferem até hoje, com raras exceções, publicar em revistas "internacionais". Até há bem pouco tempo as agências de fomento à pesquisa classificavam os periódicos em nacionais e internacionais, e os comitês científicos davam maior peso, em suas avaliações, aos trabalhos nestas últimas. Para mudar esse quadro, primeiro é necessário que se desfaçam alguns mitos. A desconstrução desses mitos é também um dos objetivos deste artigo.

\section{RESULTADOS E DISCUSSÃO}

No banco de dados ISI Web of Knowledge estão registrados 41.362 artigos científicos para o triênio 2000-2002, recuperados através da busca pelo endereço dos autores contendo a palavra "Brazil". Através do cruzamento de palavras-chave e da busca pelo nome de alguns pesquisadores brasileiros com produção científica consolidada, foi possível descortinar um panorama do que tem sido realizado no País na área de produtos naturais e selecionar 12 periódicos e sub-áreas da química de PN que serão discutidos neste artigo. Os trabalhos publicados por estes pesquisadores foram comparados com os que são publicados, por esses mesmos grupos, no Journal of the Brazilian Chemical Society e na Química Nova.

Para a análise comparativa foram selecionadas as seguintes revistas: Biochemical Systematics and Ecology, Flavour and Fragrance Journal, Fitoterapia, Journal of Agricultural and Food Chemistry, Journal of Chemical Ecology, Journal of Ethnopharmacology, Journal of Essential Oil Research, Journal of Natural Products, Phytochemistry, Phytotherapy Research, Planta Medica, e Zeitschrift fur Naturforschung $C$, pois estes foram identificados como os periódicos em que, tradicionalmente, grande parte da produção científica da comunidade química brasileira da área de produtos naturais é concentrada.

A seguir é apresentado um breve escopo das áreas de interesse de cada uma das revistas selecionadas:

Biochemical Systematics and Ecology (BSE, http:// www.elsevier.com/locate/issn/03051978): Criada principalmente para 
veicular artigos sobre sistemática micromolecular e a evolução de plantas, ampliou seu escopo de publicação nos últimos anos e hoje contém artigos sobre produtos naturais em geral, inclusive sobre determinação estrutural e ecologia. A publicação de artigos de fitoquímica convencional tem aumentado significativamente nos últimos anos. Disponível no portal de periódicos da CAPES (http:// www.periodicos.capes.gov.br)

Flavour and Fragrance Journal (FFJ, http:// www3.interscience.wiley.com/cgi-bin/jhome/4029): Revista com linha editorial similar ao Journal of Essential Oil Research, abrangendo artigos sobre fragrâncias, aromas e "flavours" de plantas e alimentos, como sugere seu título. Não disponível no portal de periódicos da CAPES.

Fitoterapia (FITO, http://www.elsevier.com/locate/fitote): A partir de 1999 passou a ser co-publicada pela Elsevier, a mesma editora da Phytochemistry. Depois dessa mudança a revista vem dando ênfase a publicação de trabalhos sobre a elucidação estrutural de produtos naturais. Além da determinação estrutural de novas substâncias naturais há neste periódico muitos artigos de atividade biológica de extratos brutos vegetais e de substâncias puras. Perfis biológicos de extratos brutos estão dentro da linha editorial da revista. A Fitoterapia ainda publica a descrição de novas ocorrências para substâncias naturais já conhecidas. Disponível no portal de periódicos da CAPES (http://www.periodicos.capes.gov.br)

Journal of Agricultural and Food Chemistry (JAFC, http:// pubs.acs.org/journals/jafcau/index.html): Com escopo bem amplo, este periódico é disponibilizado a publicações referentes à química e bioquímica de alimentos e produtos agrícolas, incluindo análise em matéria prima natural e processada, pesticidas, produtos veterinários, agentes de crescimento em plantas, fertilizantes e agroquímicos em geral, metabólitos de plantas e aspectos toxicológicos e ambientais. A revista enfoca ainda aspectos nutricionais em fitonutrientes, "flavours" e aromas. Disponível no portal de periódicos da CAPES (http://www.periodicos.capes.gov.br)

Journal of Chemical Ecology (JCE, http:// www.kluweronline.com/issn/0098-0331): Como o próprio título indica, é uma revista especializada na publicação de artigos de ecologia química em todos os seus aspectos, seja na identificação de substâncias envolvidas em processos ecológicos intra e inter-específicos em ambientes terrestres e aquáticos, assim como nas suas sínteses. Revista oficial da Sociedade Internacional de Ecologia Química (ISCE, http://www.chemecol.org), é publicada pela Kluwer Academic Publishers. Não disponível no portal de periódicos da CAPES.

Journal of Essential Oil Research (JEOR): Revista especializada na publicação de estudos sobre plantas odoríferas, em todos os aspectos analíticos e de identificação de substâncias voláteis. Não disponível no portal de periódicos da CAPES.

Journal of Ethnopharmacology (JE, http://www.elsevier.com/ locate/issn/03788741): Neste periódico são publicados desde artigos sobre atividade biológica de extratos brutos vegetais a "screenings" de extratos brutos de plantas utilizadas por determinados grupos sociais. Contém também trabalhos sobre o uso medicinal de plantas, fungos e microorganismos. Enfoca a etnofarmacologia, etnobotânica e etnoquímica. Disponível no portal de periódicos da CAPES (http://www.periodicos.capes.gov.br)

Journal of Natural Products (JNP, http://pubs.acs.org/journals/ jnprdf/index.html): criado com o nome de Loydia, vem firmando-se como a principal revista científica da área de PN desde que passou a ser publicada pela American Chemical Society (ACS). Atualmente é o principal periódico dos químicos de produtos naturais marinhos. Além da descrição de novas estruturas de PNs complexas, a linha editorial da revista tem privilegiado a publicação de artigos descrevendo a atividade biológica dos metabólitos isolados, assim como alguns artigos de desenvolvimento de metodologias (de isolamento, elucidação estrutural ou de análise de bioatividade), síntese e biotransformações de PNs. Disponível no portal de periódicos da CAPES (http://www.periodicos.capes.gov.br)

Phytochemistry (PHY, http://www.elsevier.com/locate/issn/ 00319422): Durante muito tempo foi considerado o principal periódico da área de PN. Esta era a Bíblia dos químicos de produtos naturais brasileiros. Desde 1998, no entanto, esta revista vem passando por profunda reformulação editorial diminuindo a ênfase na publicação de artigos sobre determinação estrutural de metabólitos secundários. Por outro lado, é cada vez maior o número de publicações em Phytochemistry sobre genética e biologia molecular, genômica, quimiotaxonomia e outros aspectos da bioquímica vegetal. Disponível no portal de periódicos da CAPES (http:// www.periodicos.capes.gov.br)

Phytotherapy Research (PR, http://www.wiley.com/WileyCDA/ WileyTitle/productCd-PTR.html): Nesta revista são publicados trabalhos de natureza interdisciplinar. Os trabalhos de elucidação estrutural devem vir acompanhados de resultados de atividade biológica. A publicação de "screening" de extratos brutos vegetais vem sendo desestimulada. Bioquímica e farmacologia molecular, toxicologia, patologia e aplicações clínicas de produtos naturais estão dentro do escopo editorial da revista. Um dos destaques deste periódico é a publicação de uma seção de patentes envolvendo produtos naturais. Não disponível no portal de periódicos da CAPES.

Planta Medica (PM, http://www.thieme.de/plantamedica): Atualmente é a principal revista dedicada a publicação de estudos sobre a atividade biológica de extratos vegetais e de substâncias naturais. Normalmente as exigências para publicação neste periódico são maiores do que na maioria das revistas da área de farmacologia de plantas. A publicação de novas substâncias está sempre acompanhada de estudos farmacológicos. Enfatiza estudos na área de produtos naturais bioativos e plantas medicinais, com enfoque em farmacologia, bioquímica e biologia molecular, fisiologia, biotecnologia, química de produtos naturais e métodos analíticos. Não disponível no portal de periódicos da CAPES.

Zeitschrift fur Naturforschung C - A Journal of Biosciences (ZN, http://www.znaturforsch.com/c.htm): Periódico de escopo abrangente que publica trabalhos de química em todos os seus aspectos, se estendendo da determinação estrutural de substâncias naturais, composição química de óleos essenciais à aspectos bioquímicos vegetais e genética molecular. Não disponível no portal de periódicos da CAPES.

Química Nova (QN, http://wwwquimicanova.sbq.org.br/ quimicanova.htm): Publica manuscritos nas áreas tradicionais da química incluindo ensino, história e política científica. Seu acesso é gratuito.

Journal of the Brazilian Chemical Society (JBCS, http:// jbcs.sbq.org.br): Periódico dedicado a todas as áreas da química com exceção de educação, filosofia e história. Seu acesso é gratuito.

Informações detalhadas sobre as áreas de interesse de cada uma destas revistas são encontradas nas suas respectivas páginas eletrônicas na WEB.

Neste trabalho procurou-se enfocar sub-áreas de maior representatividade da química de PN no Brasil. Isto inclui áreas tradicionais ou mais jovens, todas bem inseridas no contexto internacional. É importante ressaltar que não foram considerados artigos de síntese e de transformações de produtos naturais.

\section{Elucidação Estrutural e Isolamento de Metabólitos Secundários}

Trabalhos de determinação estrutural são feitos em todas as regiões do País. São poucos os estados brasileiros que não tem um grupo 
de pesquisa trabalhando com elucidação estrutural de produtos naturais. Há muitos bons grupos de pesquisa neste campo espalhados por todas as regiões do País, sendo o mais tradicional neste domínio o da Universidade Estadual do Norte Fluminense (UENF). Observase também uma grande coesão dos grupos de PN da UFSCar e da UNESP de Araraquara, e colaborações consolidadas do grupo de PN da UENF com os do Ceará e da UFRRJ.

A maioria dos grupos ainda concentra seu foco de pesquisa na elucidação estrutural de metabólitos secundários. O JBCS e a QN possuem um grande número de trabalhos sobre determinação estrutural de PN. Os trabalhos descrevem a análise de constituintes químicos presentes em extrato (matriz bruta, frações) em plantas das mais variadas famílias botânicas, bem como procedimentos fitoquímicos clássicos envolvendo isolamento e caracterização estrutural ${ }^{5}$.

Uma outra linha de trabalho para a identificação de PN, que vem cada vez mais tendo aceitação no Brasil, é a utilização de cromatografia gasosa na análise de extratos brutos vegetais sem a necessidade de isolamento de seus constituintes químicos. Esse domínio é muito forte na UFRJ, na USP-São Carlos e na UNESP-Araraquara. Por outro lado, a cromatografia líquida de alta eficiência está bem consolidada na UFSCar e na UNESP-Araraquara. Há também grupos com bom domínio desta técnica no Rio Grande do Sul e no Paraná. Tanto a QN como o JBCS tem um número considerável de artigos, nos quais a ferramenta de identificação de compostos naturais é a cromatografia gasosa acoplada a espectrometria de massas ${ }^{6}$.

\section{Produtos Naturais e Bioatividade}

Grupos de químicos de produtos naturais realizando trabalhos de bioatividade estão espalhados do norte ao sul do País. Basta ver os artigos publicados por pesquisadores brasileiros nos periódicos Planta Medica, Journal of Ethnopharmacology, Fitoterapia e Phytotherapy Research, no periodo de 2000 a 2002, para se ter um panorama da química de produtos naturais brasileira no domínio da bioatividade. Entretanto, pode-se afirmar que, com raras exceções, a expressiva maioria desses trabalhos são preliminares e se encerram com o próprio artigo, quando não se limitam as teses, sem os desdobramentos desejados.

Uma breve busca na literatura científica mostra que no Ceará e na Paraíba há grupos consolidados trabalhando com plantas medicinais utilizadas na medicina popular ${ }^{7}$. Nestes dois estados a associação de grupos de PN e de farmacologia é sólida e vem de longa data. A determinação estrutural acoplada com atividade biológica também está consolidada nos estados do Rio de Janeiro ${ }^{8}$, São Paulo ${ }^{9}$ e Minas Gerais ${ }^{10}$. O grupo de farmacologia de produtos naturais mais consolidado está no estado de Santa Catarina ${ }^{11}$.

No país são poucas as associações de farmacólogos e químicos de produtos naturais com mais de 10 anos. Observa-se, no entanto, que os pesquisadores mais jovens procuram de uma maneira ou de outra, quase sempre na base do improviso, realizar bioensaios simples em seus próprios laboratórios.

Para que os químicos de PN avancem no domínio da bioatividade é necessário, antes de tudo, que algumas barreiras de energia sejam vencidas. A primeira e a mais importante destas é o rompimento com a cultura da determinação estrutural que se estabeleceu na comunidade. Muitas vezes o objetivo da identificação do princípio ativo do extrato ou da fração não é alcançado porque o pesquisador encanta-se com a arquitetura molecular e se esquece do resto.

As duas palavras mais usadas hoje no Brasil pelos químicos de PN são redes de bioensaios e laboratórios virtuais. Mais importante do que essas palavras são ações que quebrem paradigmas e levem a química de produtos naturais para novos caminhos. São muito poucas, no entanto, essas ações. Vale aqui ressaltar que o edital sobre fitoterápicos do CNPq, o Programa Biota da FAPESP e o Programa Temático da FAPERJ (este último com a liberação de recursos descontinuada) são ações ainda tímidas, induzidas por estas agências de financiamento à pesquisa, que podem definir caminhos novos para a comunidade de PN. Só essas não são suficientes. Programas de bioprospecção de produtos naturais são necessários e devem ser implementados com a urgência que o tema exige. Para que esses programas sejam consistentes e frutifiquem é fundamental que haja coordenação, investimento e que deixe de ser virtual. Centrais automatizadas de bioensaios onde os equipamentos de "high throughput screening" saiam dos catálogos das empresas e sejam ligados nas tomadas da rede elétrica são indispensáveis para que a bioprospecção possa ser feita adequadamente.

Por outro lado, a comunidade química de produtos naturais brasileira deve mudar seus alvos de pesquisa. Há muitos grupos desenvolvendo pesquisas sobre atividade antioxidante, antiinflamatória, antifúngica, leishmanicida etc... Entretanto, quando os alvos são enzimas importantes envolvidas em quadros patológicos graves, que já se configuram como o grande problema de saúde da espécie humana nas próximas décadas, o número de pesquisadores envolvidos nas pesquisas com esse foco pode ser contado com os dedos de uma única mão.

\section{Produtos Naturais Voláteis}

O estudo das substâncias voláteis na área de produtos naturais passa, classicamente, pela análise de óleos essenciais de plantas obtidos por hidrodestilação e de óleos voláteis em geral, extraídos ainda pelas técnicas de headspace, fluido supercrítico e extração líquidolíquido $^{12}$

A investigação de constituintes voláteis é tradicionalmente interdisciplinar, haja vista sua expressão em áreas como a biologia floral, ecologia química, genética, ciência de alimentos, biotecnologia e agricultura. Os trabalhos nesta área são encontrados em diversos periódicos. No entanto, a análise das revistas JEOR, FFJ, JAFC e $\mathrm{ZN}$, consideradas representativas, indicam a tendência da área e a participação de grupos brasileiros no cenário internacional.

Os artigos publicados no periódico FFJ versam sobre aspectos gerais de fragrâncias e "flavours", com ênfase analítica, obtidas de produtos naturais e de alimentos. A maioria dos óleos essenciais estudados foi obtida por hidrodestilação em Clevenger. Outras técnicas de extração como "headspace", spme (microextração em fase sólida) e outras representam aproximadamente $20 \%$ do total das técnicas de extração.

A justificativa principal dos trabalhos para a seleção das plantas estudadas, está relacionada a sua utilização na medicina popular, na alimentação e na perfumaria . Há ainda estudos voltados para a variação sazonal de espécies, "chemotypes”, composição química X atividade biológica (principalmente antimicrobiana seguida de antioxidante), com enfoques tímidos em culturas de células, síntese orgânica, desenvolvimento genético e controle de autenticidade de óleos. Há um grande número de trabalhos publicados por centros de pesquisa não universitários. Em 2002-2001, 21 \% das publicações tiveram como autores os pesquisadores destes centros (indústrias e não universitários); $20 \%$ foram em parceria entre universidades e centros de pesquisas e, o restante, só de centros universitários. Os países que se destacaram em números de publicações neste periódico (2001/02), foram : India, França, Irã, Itália, Alemanha, Espanha, Brasil, destacando-se a França com o maior número de colaborações com outros países. A principal técnica de análise é a cromatografia gasosa acoplada a espectrometria de massas, apoiada pelos índices de retenção. Os dados são relatados em áreas percentuais, com pouquíssimo apoio de técnicas estatísticas. Há um número razoável 
de trabalhos descrevendo a co-injeção de padrões e, menos expressivo é o uso de RMN e IV. Um pouco tímida (4\% dos trabalhos no período) é a utilização da cromatografia gasosa multidimensional aplicada a análise quiral. Apesar de reduzidos estes trabalhos são de boa qualidade analítica. Ainda inexpressiva (embora citada em 2\% dos trabalhos) é a análise por CGAR-EM com razão isotópica. Os trabalhos publicados nos últimos 3 anos versam principalmente sobre a análise da composição química de óleos essenciais.

No periódico JEOR são publicados estudos relativos aos óleos essenciais e voláteis de plantas, exceto quanto aos aspectos agronômicos e de horticultura. Com perfil semelhante ao FFJ, a maioria dos trabalhos descreve a composição química de óleos essenciais extraídos por hidrodestilação em Clevenger e analisados por CGAREM. A ênfase em CGAR quiral é inexpressiva. A principal atividade biológica relatada é a antimicrobiana. Também há um grande número de trabalhos publicados por centros de pesquisa não universitários. Em 2002, por exemplo, 15\% das publicações tem como autores os pesquisadores destes centros (indústrias e não universitários) e $35 \%$ em parceria com universidades. Os países que se destacaram em números de publicações neste periódico (2001/02), foram : Irã, França, Brasil, Cuba, Índia, Austrália e Itália.

No JAFC, observa-se que a maioria dos artigos é referente a alimentos, cabendo ressaltar o número significativo de trabalhos analíticos em vinhos. Em relação aos óleos voláteis, são enfocadas análises de ervas, condimentos, óleos de cítricos, envolvendo estudos de condições de plantio, composição química de mesmas espécies em regiões distintas, composição química frente a diferentes processos de extração, etc. Há ainda uma fração razoável de trabalhos em voláteis $(\sim 10 \%)$ gerados por microorganismos fora dos trabalhos em vinhos.

No domínio das substâncias voláteis é expressivo o número de artigos de pesquisadores brasileiros publicados no Journal of Essential Oil Research $(\sim 10 \%)$ e no Flavour Fragrance and Journal $(\sim 8 \%)$. Por outro lado, dada a sua especificidade, este domínio é pouco representativo no JBCS e na QN. Há uma tradição no estudo de óleos essenciais no Pará, cujo grupo estava anteriormente no estado do Amazonas e no Ceará. Observa-se ainda um número significativo de artigos do Rio Grande do Sul e Ceará. Tanto na QN como no JBCS o número de trabalhos publicados sobre a determinação da constituição de óleos essenciais e de compostos voláteis, em geral, é reduzido, face ao número de artigos que são publicados por pesquisadores brasileiros no FFJ e no JEOR. Neste campo o Brasil está bem representado, até mesmo pelo potencial de sua flora odorífera, uma das mais pujantes do planeta.

A maior parte dos trabalhos publicados em Quimica Nova que discute compostos voláteis está em revisões, p. ex., óleo de copaíba ${ }^{13}$, café $^{14}$, ambergris ${ }^{15}$, reações de ozonólise em fase gasosa ${ }^{16}$ seguido de artigos em bebidas ${ }^{17}$ e constituintes de plantas em geral.

\section{Ecologia Química}

Assim como as sub-áreas já mencionadas, a de ecologia química é interdisciplinar e os trabalhos realizados podem ser encontrados em diversos periódicos incluindo aqueles específicos de zoologia, botânica e ecologia de plantas e animais em ambientes terrestres ou aquáticos. Além disso, devido a aplicação de mediadores químicos intra ou interespecíficos no controle de pragas em plantações, revistas da área agrícola também são um dos destinos para a divulgação dos trabalhos realizados por grupos de pesquisa que, normalmente, envolvem químicos e biólogos. Dependendo do ponto de vista, trabalhos de quimiossistemática ou de síntese (p. ex. síntese de feromônios de insetos) também podem ser considerados desta área. Como este artigo visa uma análise comparativa com os trabalhos publicados nas revistas da Sociedade Brasileira de Química em pro- dutos naturais, entende-se que o Journal of Chemical Ecology (JCE) é aquele com o perfil mais adequado para esta análise.

A área de Ecologia Química no Brasil é bem representada por grupos que surgiram, principalmente, através dos trabalhos pioneiros de Keith S. Brown Jr. ${ }^{18}$ e José Tércio Barbosa Ferreira ${ }^{19}$.

Hoje, com pesquisadores espalhados em vários estados brasileiros, a área já realizou três encontros nacionais (Encontro Brasileiro de Ecologia Química - EBEQ) e participou da organização do XVII Encontro Internacional de Ecologia Química (17th Annual Meeting of the International Society of Chemical Ecology, Poços de Caldas, 2000). Em decorrência deste último e do empenho de alguns pesquisadores, a revista JBCS publicou um número dedicado à Ecologia Química com 11 artigos (volume 11, número 6) que, se somados a outros publicados na mesma revista ${ }^{20}$ e comparados com aqueles publicados no JCE (todos contendo pelo menos um autor de Instituição brasileira) revelam que os trabalhos publicados no JBCS foram mais citados na literatura (1,8 citações/trabalho) do que aqueles publicados no JCE por pesquisadores brasileiros no triênio 2000-2002 (1,2 citações/trabalho).

\section{Produtos Naturais Marinhos}

Assim como em outras sub-áreas de PNs, a química dos organismos marinhos experimenta, nos últimos três anos, o crescimento e a consolidação de estudos interdisciplinares, envolvendo aquacultura, genética molecular, ecologia e aplicações biotecnológicas em geral. Apesar disso, as publicações sobre isolamento de novos produtos naturais marinhos têm se concentrado na revista Journal of Natural Products (JNP), publicada pela ACS ( http://acs.org.br ). De fato, a análise dos artigos publicados em 2000 revela que esta revista contém cerca de $42 \%$ dos trabalhos realizados, num universo de 44 revistas científicas, no que diz respeito ao isolamento de novos PNs ou à descrição de novas ocorrências para PNs já conhecidos ${ }^{21}$. Observa-se também que no triênio 2000-2002, o maior número de publicações trata do isolamento de produtos naturais de esponjas, apesar do crescente interesse em substâncias bioativas de microorganismos, onde os fungos e cianobactérias são os mais representativos. A análise das publicações do JNP, entretanto, não leva em consideração estudos sobre atividade biológica de extratos brutos de organismos marinhos, bem como trabalhos envolvendo macromoléculas naturais ou pesquisas biotecnológicas.

Apenas seis trabalhos em produtos naturais marinhos do JNP contém pelo menos um autor brasileiro e versam sobre o isolamento e a elucidação estrutural de substâncias bioativas de organismos marinhos $^{22}$. Na verdade, o número total de trabalhos publicados neste periódico por pesquisadores brasileiros é pouco expressivo: no total de cerca de 1250 trabalhos, 19 são de autoria ou co-autoria nacional.

Sem dúvida, apesar do grande potencial, a sub-área de produtos naturais marinhos é uma das menos explorada no país. No entanto, algumas publicações começam a surgir demonstrando a existência de grupos interessados neste estudo. Além dos trabalhos do JNP já mencionados, de algumas revisões ${ }^{23}$, trabalhos sobre quimiossistemática ${ }^{24}$ e atividade biológica de extratos brutos e frações semipurificadas $^{25}$, o isolamento e a atividade biológica de produtos naturais obtidos de organismos marinhos brasileiros foram publicados em outras revistas científicas no triênio em questão, cabendo ressaltar o grande número de publicações sobre polissacarídeos ${ }^{26}$.

Entre os quase 50 trabalhos sobre PN marinhos encontradas no banco de dados ISI Web of Knowledge, 8 foram publicados na revistas nacionais Química Nova (dois - uma revisão), Journal of the Brazilian Chemical Society (dois), Anais da Academia Brasileira de Ciências (duas revisões) e Brazilian Journal of Medicinal and Biological Research (dois). 
A química de produtos naturais de organismos marinhos está restrita, praticamente, aos estados do Rio de Janeiro (UFF e UFRJ) e São Paulo (USP-São Carlos). É interessante observar que o grupo da UFRJ é de bioquímicos, cujos trabalhos possuem grande impacto internacional.

Uma área de PN ainda pouco representativa é a de estudos biossintéticos, hoje limitados a dois grupos, um da UNESPAraraquara e outro da USP que, na maioria das vezes, trabalham em colaboração $0^{27}$.

\section{CONCLUSÃO}

O levantamento feito neste artigo na área de química de produtos naturais que cobre o período de 2000 a 2002 no banco de dados ISI Web of Knowledge, demonstrou que apesar da comunidade química brasileira desta área dar preferência para publicar seus artigos no exterior, o índice de citação desses artigos na literatura não difere muito, em média, da dos artigos publicados por esses mesmos pesquisadores no Journal of the Brazilian Chemical Society e na Química Nova. A variação no índice de citação depende mais do foco do trabalho do que propriamente da revista.

Outra conclusão que pode ser tirada deste artigo refere-se à qualidade das publicações. Esta independe, na maioria das vezes, da revista. Tanto o JBCS como a QN nada ficam a dever a maioria das revistas discutidas nesta publicação.

Apesar de muitos artigos terem sido publicados em periódicos diversos, a Tabela 1 é um retrato real da química de produtos naturais brasileira. Esta sub-área da química no período de 2000 a 2002 foi responsável por $12 \%$ das publicações da área de química ${ }^{28}$.

Tabela 1. Número de trabalhos publicados por periódico/ano e número de trabalhos publicados por pesquisadores brasileiros

\begin{tabular}{lrrrrr}
\hline Periódico $^{\mathrm{a}}$ & $\mathrm{II}^{\mathrm{b}}$ & $2000^{\mathrm{c}}$ & $2001^{\mathrm{c}}$ & $2002^{\mathrm{c}}$ & $\mathrm{BR}^{\mathrm{d}}$ \\
\hline BSE & 0,909 & 116 & 128 & 132 & 41 \\
FFJ & 0,639 & 73 & 85 & 97 & 19 \\
FITO & 0,589 & 141 & 155 & 107 & 41 \\
JAFC & 1,915 & 1049 & 960 & 1262 & 27 \\
JCE & 1,393 & 196 & 174 & 186 & 14 \\
JEOR & 0,368 & 185 & 152 & 185 & 48 \\
JE & 1,188 & 257 & 191 & 199 & 47 \\
JNP & 1,855 & 433 & 391 & 412 & 19 \\
PHY & 1,686 & 447 & 461 & 371 & 100 \\
PR & 0,875 & 137 & 143 & 175 & 32 \\
PM & 2,289 & 137 & 136 & 225 & 20 \\
ZN & 0,715 & 171 & 183 & 205 & 24 \\
QN & 0,637 & 133 & 134 & 134 & 48 \\
JBCS & 1,036 & 96 & 100 & 101 & 64 \\
\hline
\end{tabular}

a) BSE = Biochemical Systematics and Ecology; FFJ = Flavour and Fragance Journal FITO = Fitoterapia $; \mathrm{JAFC}=$ Journal of Agricul tural and Food Chemistry; JCE = Journal of Chemical Ecology; JEOR = Journal of Essential Oil Research; JE = Journal of Ethnopharmacology; JNP = Journal of Natural Products; PHY = Phytochemistry $; \mathrm{PR}=$ Phytoterapy Research $; \mathrm{PM}=$ Planta Medica; $\mathrm{ZN}=$ Zeitschrift fur Naturforschung $C-A$ Journal of Biosciences; $\mathrm{QN}=$ Quimica Nova; JBCS = Journal of the Brazilian Chemical Society; b)II = Indice de Impacto (fonte: ISI Web of Knowledge); c) Número total de artigos publicados no ano; d) Número de trabalhos com pelo menos um autor de Instituição brasileira na área de PN.
Apesar dos números serem expressivos como pode ser constatado pela leitura atenta deste artigo, os químicos de produtos naturais brasileiros precisam romper as paredes celulares da biologia. A descoberta de um princípio ativo, por mais importantes que sejam as suas propriedades farmacológicas, pode ficar restrita a uma publicação científica se o seu isolamento pelas técnicas químicas tradicionais for economicamente inviável. Por isso, o químico de produtos naturais deve enveredar pelos estudos de biossíntese, melhoramento genético de plantas medicinais, filogenia molecular, clonagem de enzimas e por domínios da biologia molecular. Se o não fizer vai perder o bonde da história.

Mais uma vez o Professor Otto R. Gottlieb (http:// www.prossiga.br/ottogottlieb) nos mostra com suas publicações e sapiência que há luz no fim do túnel.

\section{REFERÊNCIAS}

1. Pinto, A. C.; Peixoto, E. M. A.; Galembeck, F.; de Andrade, J. B.; de Paoli, M. A.; Avaca, L. A.; Alves. O. L.; Quim. Nova 1997 (número especial), 20,81 .

2. Gibbs, W. W.; Sci. Am. 1995, 273, 92.

3. Guimarães, L. M. P.; Tese de Doutorado; Universidade de São Paulo (FFLCH), Brasil, 1994.

4. Pinto, A. C.; Silva, D. H. S.; Bolzani, V. S.; Lopes, N. P.; Epifanio, R. de A.; Quim. Nova 2002, 25, 45.

5. Ver como exemplo as referências mais recentes: Bastos, A. B. F.D'O.; de Carvalho, M. G.; Velandia, J. R.; Braz-Filho, R.; Quim. Nova 2002, 25, 241; Pereira, A. S.; Seixas, F. R. M. S.; Aquino Neto, F. R.; Quim. Nova 2002, 25, 321; Pauletti, P. M.; Araújo, A. R.; Bolzani, V. S.; Young, M. C. M.; Quim. Nova 2002, 25, 349; Maciel, M. A. M.; Pinto, A. C.; Veiga Jr., V. F.; Grynberg, N. F.; Echevarria, A.; Quim. Nova 2002, 25, 429; Silva, K. L.; Cechinel Filho, V.; Quim. Nova 2002, 25, 449; Ribeiro, C. V. C.; Kaplan, M. A. C.; Quim. Nova 2002, 25, 533; Paiva, S. R.; Fontoura, L. A.; Figueiredo, M. R.; Mazzei, J. L.; Kaplan, M. A. C.; Quim. Nova 2002, 25, 717; de Vasconcelos, A. F. F.; Godinho, O. E. S.; Quim. Nova 2002, 25, 1057; de Oliveira, M. C. C.; de Carvalho, M. G.; da Silva, C. J.; Werle, A. A.; J. Braz. Chem. Soc. 2002, 13, 119; Pires, V. S.; Taketa, A. T. C.; Gosmann, G.; Schenkel, E. P.; J. Braz. Chem. Soc. 2002, 13, 135; Paula, V. F.; Barbosa, L. C. A.; Errington, W.; Howarth, O. W.; Cruz, M. P.; J. Braz. Chem. Soc. 2002, 13, 276; Monteiro, V. F. F.; Mathias, L.; Vieira, I. J. C.; Schripsema, J.; Braz-Filho, R.; J. Braz. Chem. Soc. 2002, 13, 281; Branco, A.; Pinto, A. C.; Ifa, D. R.; Braz-Filho, R.; J. Braz. Chem. Soc. 2002, 13, 318; Suarez, L. E. C.; Menichini, F.; Monache, F. D.; J. Braz. Chem. Soc. 2002, 13, 339; Amaro-Luis, J. M.; Ramírez, I.; DelgadoMéndez, P.; Jorge, Z. D.; J. Braz. Chem. Soc. 2002, 13, 352; Pupo, M. T.; Adorno, A. T.; Vieira, P. C.; Fernandes, J. B.; da Silva, M. F. G. F.; Pirani, J. R.; J. Braz. Chem. Soc. 2002, 13, 382; Nogueira, R. T.; Giacomini, R. A.; Shepheerd, G. J.; Imamura, P. M.; J. Braz. Chem. Soc. 2002, 13, 389; Barreiros, M. L.; David, J. M.; Pereira, P. A. P.; Guedes, M. L. S.; David, J. P.; J. Braz. Chem. Soc. 2002, 13, 669; Cruz, F. G.; Moreira, L. M.; Santos, N. A. S.; Guedes, M. L. S.; J. Braz. Chem. Soc. 2002, 13, 704; Gottlieb, O. R.; Borin, M. R. M. B.; J. Braz. Chem. Soc. 2002, 13, 772.

6. Pinto, A. C.; Braga, W. F.; Rezende, C. M.; Garrido, F. M. S.; Veiga Jr., V. F.; Bergter, L.; Patitucci, M. L.; Antunes, O. A. C.; J. Braz. Chem. Soc. 2000, 11, 355; Negri, G.; Marcucci, M. C.; Salatino, A.; Salatino, M. L. F.; J. Braz. Chem. Soc. 2000, 11, 453; Pereira, A. S.; Aquino Neto, F. R.; Quim. Nova 2000, 23, 370; Valente, A. L. P.; Augusto, F.; Pérez, E. R.; Cardoso, D. R.; Franco, D. W.; Keukeleire, D. D.; Quim. Nova 2001, 24, 10; De Maria, C. A. B.; Moreira, R. F. A.; Quim. Nova 2001, 24, 516; Branco, A.; Pizzolatti, M. G.; Quim. Nova 2002, 25, 15.

7. Guedes, M. M.; Cunha, A. N.; Silveira, E. R.; Rao, V. S. N.; Planta Med. 2002, 68, 1044; Guedes, D. N.; Silva, D. F.; Barbosa, J. M.; Medeiros, I. A.; Planta Med. 2002, 68, 700 .

8. Grynberg, N. F.; Carvalho, M. G.; Velandia, J. R.; Oliveira, M. C.; Moreira, I. C.; Braz-Filho, R.; Echevarria, A.; J. Med. Biol. Res. 2002, 35, 819. da Silva, C. A.; Parente, J. P.; Planta Med. 2002, 68, 74. Almeida, A. P.; da Silva, S. A. G.; Souza, M. L. M.; Lima, L. M. T. R.; Rossi-Bergmann, B.; Moraes, V. L. G.; Costa, S. S.; Planta Med. 2000, 66, 134.

9. Vieira, P. C.; Mafezoli, J.; Pupo, M. T.; Fernandes, J. B.; da Silva, M. F. G. F.; de Albuquerque, S.; Oliva, G.; Pavão, F.; Pure Appl. Chem. 2001, 73, 617. da Silva, R. V.; Navickiene, H. M. D.; Kato, M. J.; Bolzani, V. D. S.; Meda, C. I.; Young, M. C. M.; Furlan, M.; Phytochemistry, 2002, 59, 521. 
10. Cortes, S. F.; Valadares, Y. M.; de Oliveira, A. B.; Lemos, V. S.; Barbosa, M. P. T.; Braga, F. C.; Planta Med. 2002, 68, 412.

11. Niero, R.; Alves, R. V.; Cechinel, V.; Calixto, J. B.; Hawkes, J. E.; Sant'Ana, A. E. G.; Yunes, R. A.; Planta Med. 2002, 68, 850.

12. Monsandl, A.; J. Chromatogr. 1992, 624, 267; Bartak, P.; Bednar, P.; Cap, L.; Ondrakova, L.; Stransky, Z.; J. Sep. Sci. 2003, 26, 715; Knudsen, J. T.; Tollsten, L.; Bot. J. Linn. Soc. 1993, 113, 263; Stashenko, E.E.; Cervantes, M.; Combariza, Y.; Fuentes, H.; Martinez, J.R.;HRS- J. High Res. Chrom. 1999, 22, 343; Marriott, P.J.; Shellie, R.; Cornwell, C.; J. Chromatogr. A 2001, 936, 1; Bartak, P.; Bednar, P.; Cap, L.; Ondrakova, L.; Stransky, Z.; J. Sep. Sci. 2003, 26, 715.

13. Veiga Jr., V. F.; Pinto, A. C.; Quim. Nova 2002, 25, 273.

14. Moreira, R. F. A.; Trugo, L. C.; De Maria, C. A. B.; Quim. Nova 2000, 23, 195;

15. Tenius, B. S. M.; Schroeder, E. K.; Schenato, R. A.; Quim. Nova 2000, 23,225 ;

16. Nunes, F. M. N.; Pereira, P. A. P.; de Andrade, J. B.; Quim. Nova 2000, 23, 794 .

17. Andrade-Sobrinho, L. G.; Boscolo, M.; Lima-Neto, B. S.; Franco, D. W.; Quim. Nova 2002, 25, 1074.

18. Ver como exemplo: Brown Junior, K. S.; Trigo, J. R.; Chemoecology 1996, $5-6,119$.

19. Ver como exemplo:Ferreira, J. T. B.; Zarbin, P. H. G.; Química Nova na Escola 1998, 7, 3.

20. House, P. E.; do Nascimento, R. R.; J. Braz. Chem. Soc. 2001, 12, 196; Cintra, P.; Malaspina, O.; Petacci, F.; Fernandes, J. B.; Bueno, O. C.; Vieira, P. C.; da Silva, M. F. G. F.; J. Braz. Chem. Soc. 2002, 13, 115.

21. Faulkner, D. J.;Nat. Prod. Rep. 2002, 19, 1

22. Braekman, JC; Daloze, D.; Tavares, R.; Hadju, E.; Van Soest R. W. M.; J. Nat. Prod. 2000, 63, 193; Torres, Y. R.; Berlinck, R. G. S.; Magalhães, A.; Schefer, A. B.; Ferreira, A. G.; Hadju, E.; Muricy, G.; J. Nat. Prod. 2000, 63, 1098; Maia, L. F.; Epifanio, R.de A.; Fenical, W.; J. Nat. Prod. 2000, 63, 1427; Britton, R.; de Oliveira, J. H. H. L.; Andersen, R. J.; Berlinck, R. G. S.; J. Nat. Prod. 2001, 64, 254; Davyt, D. ; Fernandez, R. ; Suescun, L.; Mombru, A. W.; Saldana, J.; Dominguez, L.; Coll, J.; Fujii, M. T.; Manta, E.; J. Nat. Prod. 2001, 64, 1552; B. M. ; Granato, A. C.; Berlinck, R. G. S.; Magalhaes, A.; Schefer, A. B.; Ferreira, A. G. ; Pinheiro, U. S. ; Hajdu, E.; J. Nat. Prod. 2002, 65, 796.

23. Carvalho, L. R. ; Roque, N. F.; Quim. Nova 2000, 23, 757-764 Schwartsmann, G.; Ann. Oncol. 2000, 11, 235, Suppl. 3; Berlinck, R. G S.; Nat. Prod. Rep. 2002, 19, 617; Kelecom, A.; An. Acad. Bras. Cienc. 2002, 74,151; Pavão, M. S. G.; An. Acad. Brasil. Ciênc. 2002, 74, 105.
24. Teixeira, V. L.; Cavalcanti, D. N.; Pereira, R. C.; Biochem. System. Ecol. 2001, 29, 313; De-Paula, J. C.; Pedrini, A. D.; Pinheiro, M. D.; Pereira, R. C.; Teixeira, V. L.; Biochem. System. Ecol. 2001, 29, 425.

25. Lagos, P.; Duran, R.; Cervenansky, C.; Freitas, J. C.; Silveira, R.; Braz. J. Med. Biol. Res. 2001, 34, 895; Rangel, M.; de Sanctis, B.; de Freitas, J. C.; Polatto, J. M.; Granato, A. C.; Berlinck, R. G. S.; Hajdu, E.; J. Exp. Mar. Biol. Ecol. 2001, 262, 31; Da Gama, B. A. P.; Pereira, R. C. ; Carvalho, A.G. V.; Coutinho, R.; Yoneshigue-Valentin, Y.; Biofouling 2002, 18, 13; Monks, N. R.; Lerner, C.; Henriques, A. T.; Farias, F. M.; Schapoval, E. E. S.; Suyenaga, E. S.; da Rocha, A. B.; Schwartsmann, G.; Mothes, B.J. Exp. Mar. Biol. Ecol. 2002, 281,1.

26. Ver como exemplo: .Granato, A. C.; Berlinck, R. G. S.; Magalhães, A.; Schefer, A. B.; Ferreira, A. G.; de Sanctis, B.; de Freitas, J. C.; Hajdu, E.; Migotto, A. E.; Quim. Nova 2000, 23, 594; Pereira, R. C.; Cavalcanti, D. N.; Teixeira, V. L.; Mar. Ecol. Prog. Ser. 2000, 205, 95; Epifanio, R. de A.; Maia, L. F.; Fenical, W.; J. Braz. Chem. Soc. 2000, 11, 584; Zierer, M. S.; Mourão, P. A. S.; Carbohydr. Res. 2000, 328, 209; Veervort, H.; Fenical, W.; Epifanio, R. de A. J. Org. Chem. 2000, 65, 782; Cinel, B.; Roberge, M.; Behrisch, H.; van Ofwegen, L.; Castro, C. B.; Andersen, R. J.; Org. Lett. 2000, 2, 257; Kaiser, C. R.; Pitombo, L. F.; Pinto, A. C.; Magn. Res. Chem. 2001, 39, 147; Kerchove, C. M. ; Markus, R. P. ; Farias, W. R. L.; Nazareth, R. A.; Mourao, P. A. S.; Tromb. Haemostasis 2001, 86, 1540; Freitas, J. C.; Costa-Lotufo, L. V.; Braz. J. Med. Biol. Res. 2002, 35, 485; Torres, Y. R.; Berlinck, R. G. S.; Nascimento, G. G. F.; Fortier, S. C.; Pessoa, C.; de Moraes, M. O.; Toxicon 2002, 40, 885; Torres, Y. R. ; Bugni, T. S. ; Berlinck, R. G. S. ; Ireland, C. M.; Magalhães, A.; Ferreira, A. G.; da Rocha, R. M.; J. Org. Chem. 2002, 67, 5429; Coutinho, A. F.; Chanas, B.; Souza, T. M. L. E.; Frugrulhetti, I. C. P. P.; Epifanio, R. de A.; Heterocycles 2002, 57, 1265; Tapon-Bretaudiere, J.; Chabut, D.; Zierer, M.; Matou, S.; Helley, D.; Bros, A.; Mourão, P. A. S.; Fischer, A. M.; Mol. Cancer Res. 2002, 1, 96; Santos, V. L. C. S.; Billett, D. S. M.; Wolff, G. A.; J. Braz. Chem. Soc. 2002, 13, 653.

27. Corsino, J.; de Carvalho, P. R. F.; Kato, M. J.; Latorre, L. R.; Oliveira, O. M. M. F.; Araújo, A. R.; Bolzani, V. D.; Franca, S. C.; Pereira, A. M. S.; Furlan, M.; Phytochemistry 2000, 55, 741.

28. da Gama, A. A. S.; Cadore, S.; Ferreira, V. F.; Quim. Nova 2003, 26, 618. 